\title{
MACHINE STUDY AND IMPROVEMENT OF THE HLS STORAGE RING
}

\author{
Y. Jin*, Z.Liu, W.Li, Y.Li, A.Zhou, W.Zhang, Q.Jia, B. Sun,_NSRL, USTC, Hefei, China
}

\section{Abstract}

The machine studies and improvements for the HLS storage ring are presented here. Since 1993 some beam instabilities such as head-tail instability and bunch lengthening as well as beam-cavity interaction have been observed and studied. The ion-trapping phenomenon is being studied. An RF knock out system for single bunch or for non-uniform filling to get high beam current has been installed into the ring and got some good results. A superconducting wiggler of 6 Tesla and an optical klystron (OK) used for FEL experiments as two insertion devices have been installed into the HLS. The wiggler has been commissioned and got more than $100 \mathrm{~mA}$ beam current. In order to make further improving performance of the machine, a Phase-II Project is going on.

\section{INTRODUCTION}

The HLS (Hefei Light Source) is a second generation synchrotron radiation source. It consists of an $800 \mathrm{MeV}$ storage ring and a $200 \mathrm{MeV}$ linac injector. It was constructed in 1989 and the first stored beam was got on April 26, 1989. The machine commissioning was completed in June 1991[1] . The main body of the HLS is a storage ring. The lattice of the storage ring is TBA structure[2]. It is a separated function strong focusing type. The circumference of the ring is 66.1308 meters. Its critical wavelength is $24 \AA$. The harmonic number is 45 . There are four periods with four long straight sections of $3.36 \mathrm{~m}$ each. A superconducting wiggler of 6 Tesla has been installed in the long straight section that the RF cavity had occupied. An optical klystron has also been installed in the another long straight section of the ring.

The lattice of the storage ring has two different operating configurations: (1) GPLS (General Purpose Light Source); (2) HBLS (High Brilliance Light Source). The configuration of GPLS is in routine operation now; the HBLS is a low emittance configuration $\left(\varepsilon_{\mathrm{x}}=13.5 \mathrm{~nm}\right)$, because of some difficulties for injection, is not in operation yet. But after the Phase-II project, the injection system will be rebuilt, the HBLS configuration will be put in operation.

\section{MACHINE STUDIES}

We have carried out some beam instabilities research in HLS storage ring. It includes head-tail instability, bunch

*Email: jin@ustc.edu.cn lengthening and energy spread widening, beam-cavity interaction, etc. The ion trapping is being studied.

\subsection{Head-tail Instability}

The current threshold of the head-tail instability of the HLS storage ring that calculated by Sacherer formula is about $40 \mu \mathrm{A}$ per bunch only, but actual current threshold of head-tail instability in HLS storage ring that measured is about $2.1 \mathrm{~mA}$ per bunch. The experimental value is about 50 times larger than the theory value. After researched, we found that the clearing electrodes existing in the vacuum chamber played the role of the Landau damping[3]. There are 17 clearing electrodes distributed along the vacuum chamber of the whole storage ring in order to eliminate the ion trapping. They are added DC voltage of 800-1000 volts. They will produce an electric field of dipole, quadrupole, sextupole, octupole, etc. The octupole component would produce a tune spread about $1 \times 10^{-3}$ in the betatron oscillation of the particles in a bunch. The tune spread will stabilize the beam by Landau damping, and increase the current threshold of the head-tail instability.

\subsection{Bunch Lengthening and Energy Spread Widening}

The bunch lengthening and energy spread widening were observed in the HLS storage ring. The bunch length was measured by a digital oscilloscope of $20 \mathrm{GHz}$. The energy spread was measured by decreasing the longitudinal acceptance via lowering the RF voltage until the longitudinal quantum lifetime becomes dominant. Then we can get the energy spread from measured quantum lifetime and the height of the RF bucket. The experiment results show that as the current increase from several $\mathrm{mA}$ to $130 \mathrm{~mA}$, the bunch length $\left(\sigma_{\mathrm{s}}\right)$ has a lengthening from $3.8 \mathrm{~cm}$ to $10.33 \mathrm{~cm}$. The experiment results also show that the energy spread widening occurred at about $0.2 \mathrm{~mA}$ per bunch, which is the current threshold of the longitudinal instability. While the beam current reached $2 \mathrm{~mA}$ per bunch, the energy spread from $4.6 \times 10^{-4}$ goes up to $5 \times 10^{-4}[4]$.

\subsection{Beam-Cavity Interaction}

Research of the beam-cavity interaction in the HLS storage ring has gotten some progress. Two kinds of longitudinal oscillation of the beam were observed in HLS storage ring. The frequency of the first one is 875.11 MHz, and the second one is $1541 \mathrm{MHz}$. They are 
excited by the two HOMs (High Order Modes) of the $\mathrm{TM}_{012}$ and $\mathrm{TM}_{032}$ of the RF cavity respectively [4]. The storage ring working at $800 \mathrm{MeV}$, we did not observe beam loss due to the above two longitudinal oscillations. But in the case of injection at $200 \mathrm{MeV}$, we found the longitudinal oscillation induced by $\mathrm{TM}_{012}$ mode is dependent on the tuner position. If the tuner position is at $25.4 \mathrm{~mm}$, the longitudinal oscillation of frequency $875.11 \mathrm{MHz}$ is so strong that the beam accumulating is very difficult, and energy ramping from $200 \mathrm{MeV}$ to 800 $\mathrm{MeV}$, the beam may be lost. In this case, we can keep the tuner position off $25.4 \mathrm{~mm}$, and the injection has no problem.

\subsection{Ion Trapping Phenomenon}

The ion trapping is being studied. It causes tune shift of betatron oscillation and beam blow-up in transverse direction. The tune shift was measured for beam current of $100 \mathrm{~mA}$ as $\Delta v=0.0015$ for vertical direction and corresponding neutralization factor $\eta_{\mathrm{i}}$ is $0.6 \%$. There are two types of ion clearing electrodes in the storage ring. One of them is DC clearing electrode used to overcome attraction of beam potential to ions, the other is RF clearing electrode used to shake ions away from beam orbit. The measurement shows that the minimum clearing voltage is about $350 \mathrm{~V}$ and the corresponding electric field is $46 \mathrm{~V} / \mathrm{cm}$ for DC clearing electrode (for beam current of $77 \mathrm{~mA}$ ). For RF clearing electrode, the best shaking frequency lies in 1.4-1.6 $\mathrm{MHz}$ range for $\mathrm{CO}^{+}$ions. In addition, we found that the partial-filling injection modes can also overcome ion trapping. This study is being continued.

\section{MACHINE IMPROVEMENTS}

\subsection{Miscellaneous Improvements}

We have made a beam profile monitor of the storage ring, in order to measure beam cross-section and beam emittance. The monitor is composed of an imaging optical system and CCD TV camera. The image of the profile is displayed in the central control room [5]. We remade the cooling water loop for the bending magnets so that the local over-heating phenomena have disappeared. We have made a RF knock out (RFKO) system for single bunch research. The Hefei machine is usually operating at the multiple bunches configuration. There are totally 45 bunches in the storage ring. In order to satisfy requirements of time-resolved spectroscopy experiments and machine studies, the storage ring must operate in different configurations of single bunch, 3 bunches, 5 bunches, etc. From 1994, the R \&D of an RF knock out (RFKO) system began. A prototype of RFKO has been completed now. Preliminary test has been carried out. The current of single bunch reached $30 \mathrm{~mA}$ in the storage ring. Using the RFKO system can achieve to fill a part of the buckets to avoid multi-bunch instability and increase beam current. The maximum current reached $350 \mathrm{~mA}$.

The other up-grade and improvement plans will put in the phase-II project.

\subsection{Superconducting Wiggler}

In order to extend the spectrum range of HLS from VUV to hard $\mathrm{X}$ ray, a $6 \mathrm{~T}$ superconducting wiggler of one period with three poles was designed in 1994-1995, constructed in 1996, completed in 1997. It includes a main body of superconducting wiggler magnet and cryostat system, power supplies system, control system, helium gas recovery system, special vacuum chamber, etc. All of them were completed at the end of 1997. In the beginning of 1998, the wiggler was installed into the HLS storage ring. In March of 1998, the wiggler was preliminary commissioned with beam, and got a beam of more than $100 \mathrm{~mA}$ at $800 \mathrm{MeV}$ in the storage ring. The peak value of the magnetic field of the wiggler reached 6.04 Tesla. The magnetic field ramp of the wiggler from zero to 6 Tesla spent 6 minutes, the rise speed is 1 $\mathrm{T} / \mathrm{min}$, and did not occur quench phenomenon. From October to December 1998, the superconducting wiggler and the XAFS beam line as well as experimental station were commissioned in combination and tested for 45 days, with the wiggler of 6 Tesla in successful operation. The specification of the wiggler is listed in table 1 .

Table 1 Specification of the superconducting wiggler

\begin{tabular}{|l|l|l|}
\hline Specification & Designed & Measured \\
\hline Peak field & 6 Tesla & 6.04 Tesla \\
\hline Main current & $460 \mathrm{~A}$ & $462 \mathrm{~A}$ \\
\hline Compensation current & $<20 \mathrm{~A}$ & $<5 \mathrm{~A}$ \\
\hline Field wavelength & $200 \mathrm{~mm}$ & $188 \mathrm{~mm}$ \\
\hline Beam tube size $(\mathrm{H} \times \mathrm{V})$ & $90 \times 24 \mathrm{~mm}^{2}$ & $90 \times 24 \mathrm{~mm}^{2}$ \\
\hline Ramping rate & $1 \mathrm{~T} / \mathrm{min}$ & $1 \mathrm{~T} / \mathrm{min}$ \\
\hline Critical wavelength & $4.85 \AA$ & $4.82 \AA$ \\
\hline
\end{tabular}

The main body of the superconducting wiggler magnet with cryostat was manufactured by Kurchatov Institute in Russia. The coils of the wiggler were made of Nb-Ti. The liquid He consumption is 25-30 liters per day. The performance of the wiggler satisfies design requirement. The wiggler is located on a long straight section at the down stream of the RF cavity. It will induce tune $\left(v_{x}, v_{y}\right)$ shift and closed orbit distortion as well as dynamic aperture reduction. The calculation results show that the $\beta_{\text {ymax }}$ will rise from $13 \mathrm{~m}$ to $32 \mathrm{~m}$ and $v_{\mathrm{y}}$ will shift from 2.58 to 2.678 , that is near the $1 / 3$ resonant line. The particle tracking shows that the dynamic aperture has been changed so much that injecting and storing beam is impossible. Therefore the lattice parameters must be compensated [6]. During the preliminary commissioning, we adjust two sets of 
quadrupole to reduce the $v_{\mathrm{y}}$ from 2.68 to 2.61 to avoid the $1 / 3$ resonant line.

\subsection{FEL}

A pure permanent magnets Optical Klystron (OK) has been designed and constructed for Storage Ring FEL. It will be used for the coherent harmonic generation $(\mathrm{CHG})$ experiments firstly. The total length of the OK is 1.944 $\mathrm{m}$. It consists of three sections, one dispersion section and two undulator sections. Each undulator section is consists of 12 periods with period length $72 \mathrm{~mm}$. The two undulator sections are identical. The magnetic field is 0.3 Tesla with gap $40 \mathrm{~mm}$. Between the two undulator sections is a dispersion section of $216 \mathrm{~mm}$, one period. The magnetic field of the dispersion section is 0.6 Tesla with gap $40 \mathrm{~mm}$. They are assembled in a frame. The gap of three sections can be adjusted independently from $36 \mathrm{~mm}$ to $140 \mathrm{~mm}$. The Optical Klystron has been installed into the storage ring and commissioned. The experiments of spontaneous radiation have been carried out. The measured modulation factor of the spontaneous radiation spectrum is increased from 0.236 initially to 0.76 . The further more work will be continued.

\section{PHASE-II PROJECT}

The Hefei Light Source was built in 1989. A series of important experiments have been completed at five stations, but some defects of the machine have also been exposed. In addition, the five existing beamlines and five experiment stations are obviously insufficient for such a dedicated light source, so a phase-II project was proposed in 1994. The main contents of the project are rebuilding and improving some parts or equipment of the machine, and adding 8 beamlines and 8 experiment stations. This project was approved by the government in 1996. It has been put into practice from FY 1997 and finished by 2001. The total budget is about US $\$ 14$ million. The main contents of the phase-II project include:

\subsection{Machine Improvement}

(1) RF system: Make a new RF cavity and a new RF power supply. The cooling loop of the new cavity will be more reasonable and the frequency tuning capability will be larger than the old one. The RF power of the new transmitter will be larger than the old one.

(2) Injection equipment: Use thyratrons for pulse switch of the kicker power supply instead of the spark gap of the old one. Put four kickers in the same straight section for injection instead of the three kickers in the three straight sections of the former injection scheme.

(3) Power supplies for magnets: There are 180 DC power supplies for the magnets of the storage, transport line and linac. 40-50 DC power supplies need to be replaced with new ones, because some of them are not stable, and some of them are at high fault rate.
(4) Vacuum chamber of the storage ring: Nonevaporable Getters (NEG) will be put in the downstream of each bending vacuum chamber to reduce dynamic pressure gradient of the storage ring vacuum chamber. Use hydraulic pressured bellows instead of the welded bellows. Increase vacuum monitoring points.

(5) Control system and beam diagnostic: The original control system has no shared database and has little communication between microcomputers. The goal of updating this system is set up a local network consisting of two working stations, some servers and PCs. Set up a database. Improve beam diagnostic system, and enhance its function of failure judgment.

(6) Linac: Use a $3 \mathrm{GHz}$ solid microwave source to drive high power klystron instead of the medium power klystron. Improve the $\mathrm{I} / \Phi / \mathrm{A}$ unit, and improve the energy monitor and feedback system, etc.

(7) Undulator: Plan to set up a permanent magnet undulator as an insertion device. Its wavelength range will be from $100 \AA$ to $2000 \AA$. The brilliance of the undulator radiation will be higher than the bending magnet radiation by two to three orders.

\subsection{Construction of 8 New Beamlines and 8 New Experiment Stations}

(1) Surface Physics (with angle-resolved photoemission);

(2) X-ray diffraction and scattering;

(3) LIGA technique;

(4) Atomic and molecular physics;

(5) Photo-acoustic and Photo-thermal spectroscopy;

(6) Infrared and far infrared spectroscopy;

(7) Soft X-ray magnetic circular dichroism;

(8) Spectral radiation standard and metrology.

\section{REFERENCES}

[1] Y. Jin, Status of the Hefei Synchrotron Radiation Source, Proceedings of The Third Joint Symposium on Synchrotron Radiation Sources (Nov. 1992, Korea).

[2] Y.Jin, Z. Liu et al, The Magnet Lattice of the Storage Ring for HESYRL, Proceedings of the International Conference on Synchrotron Radiation Applications (1989, Hefei, China).

[3] L. Chen, B. Sun, J. Wang, Y. Jin, Single Bunch Instabilities in HLS Ring, Proceedings of the Workshop on Beam Instabilities in Storage Rings (July 1994, Hefei, China).

[4] J. Dai et al, Study of Multiple Bunch Instability Oscillations for Hefei Synchrotron Radiation Storage Ring, Journal of China University of Science and Technology, Vol.27, No.4 (1997).

[5] Z. Fang, G. Wang et al, Monitoring the Beam Profile in HLS with Synchrotron Light, N.I.M in Physics Research A 370 (1996).

[6] N. Liu, Y. Jin, Z. Liu, The Influence of Superconducting Wiggler on HLS Storage Ring Lattice Parameters and Its compensation, Proceedings of The Sixth China-Japan Joint Symposium on Accelerators for Nuclear Science and Their Applications (Oct.1996, Chengdu, China). 